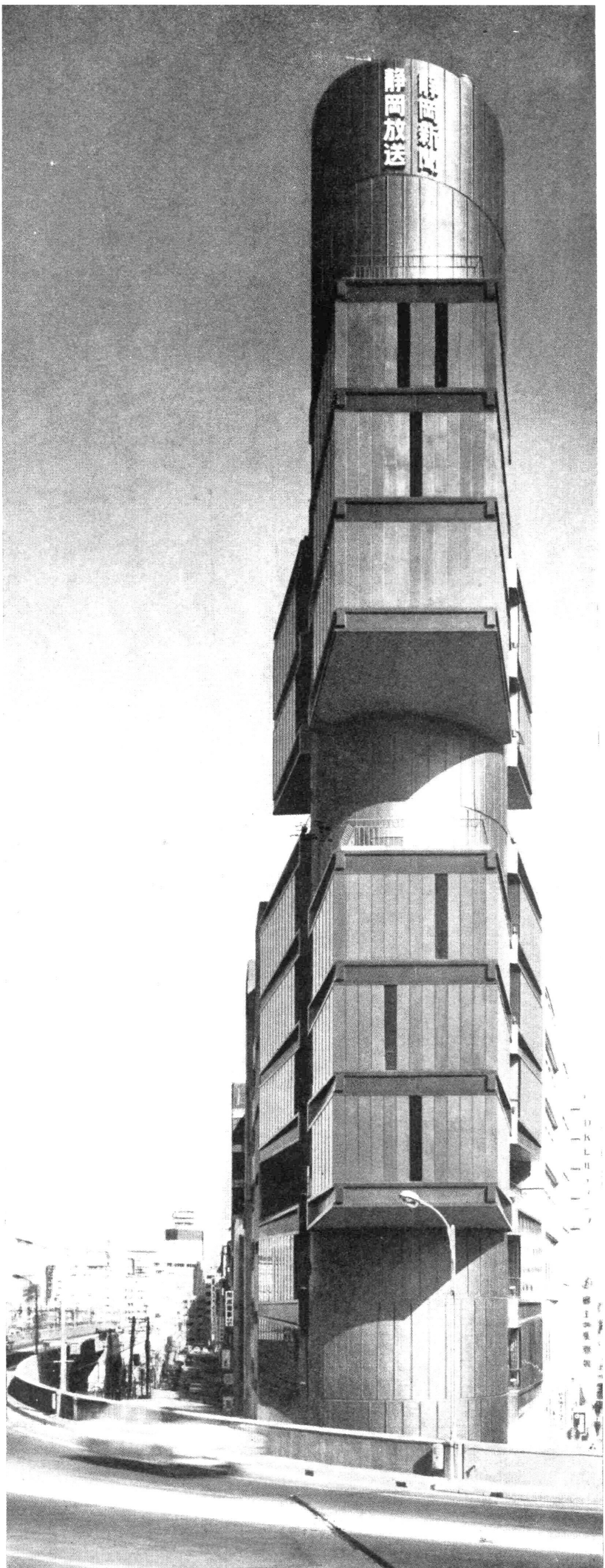

\title{
difusora de TV y diario de Shizuoka TOKYO
}

KENZO TANGE, arquitecto, y colaboradores Estructura: Laboratorio SHIGERU AOKI

\section{simopsis}

El edificio está organizado alrededor de un eje vertical constituido por una columna central -de $7,70 \mathrm{~m}$ de diámetro y $57 \mathrm{~m}$ de altura-, a base de estructura mixta - metálica y de hormigón armado-, y que consta de: sótano y doce plantas de altura.

No obstante las extremadamente reducidas dimensio nes del solar, el edificio «destaca» del conjunto urbano y sirve como punto de referencia a los viajeros que acceden al centro de Tokyo procedentes de las autopistas o del super-expreso Shinkauzen (el más rápido del mundo).

Este edificio, sede en Tokyo de la difusora de TV y del Diario de Shizuoka, ha sido construido en el distrito de Ginza, disfruta de un emplazamiento privilegiado y además, se puede llegar cómodamente hasta él, caminando desde los hoteles más céntricos de la ciudad.

Ginza es una zona proverbialmente tradicional de la capital japonesa; los caracteres chinos de "Ginza» señalan que en ella se acuñaban antiguamente las monedas de plata.

Su desarrollo comenzó después del catastrófico terremoto de 1923. Los mejores negocios de Tokyo se establecieron aquí y, como consecuencia, en ellos el comprador podía encontrar lo más selecto de cada artículo y sus calles se popularizaron. Desde entonces hasta hoy, el pasearse por sus aceras se convirtió en un pasatiempo habitual de los habitantes de Tokyo. Ello motivó que el valor del terreno subiera vertiginosamente - señalemos que los precios más altos del Japón, país ya notorio por el costo de sus solares, se paga en Ginza-. Por estas razones, aunque el terreno sea estrecho, su posición posee un significativo potencial. 

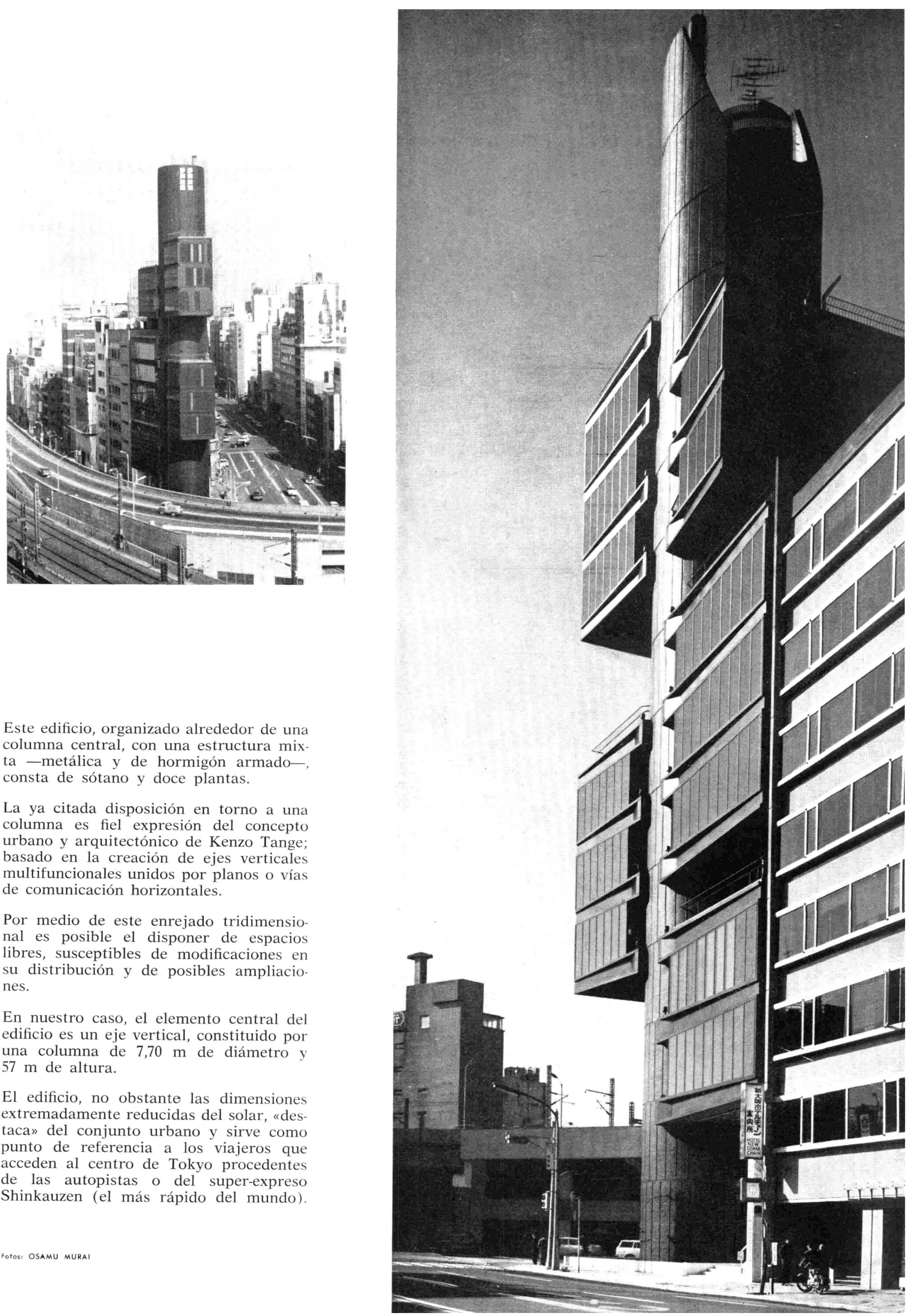

Este edificio, organizado alrededor de una columna central, con una estructura mix ta -metálica y de hormigón armadoconsta de sótano y doce plantas.

La ya citada disposición en torno a una columna es fiel expresión del concepto urbano y arquitectónico de Kenzo Tange: basado en la creación de ejes verticales multifuncionales unidos por planos o vías de comunicación horizontales.

Por medio de este enrejado tridimensional es posible el disponer de espacios libres, susceptibles de modificaciones en su distribución y de posibles ampliaciones.

En nuestro caso, el elemento central del edificio es un eje vertical, constituido por una columna de $7,70 \mathrm{~m}$ de diámetro y $57 \mathrm{~m}$ de altura.

El edificio, no obstante las dimensiones extremadamente reducidas del solar, «destaca» del conjunto urbano y sirve como punto de referencia a los viajeros que acceden al centro de Tokyo procedentes de las autopistas o del super-expreso Shinkauzen (el más rápido del mundo). 
1. Oficina.-2. Aseo.-3. Conductos.-4. Jardinera. 5. Vestíbulo de ascensores.-6. Cocina.-7. Café. 8. Accesos. - 9. Entrada.-10. Estanque.-11. Depósito de aceite.-12. Aire acondicionado--13. Depósito de agua.-14. Generadores.-15. Transformador.-16. Maquinaria ascensor.-17. Jardín.-18. rencias.-21. Nivel del paso elevado.-22. Equipo eléctrico.-23. Cimentación.
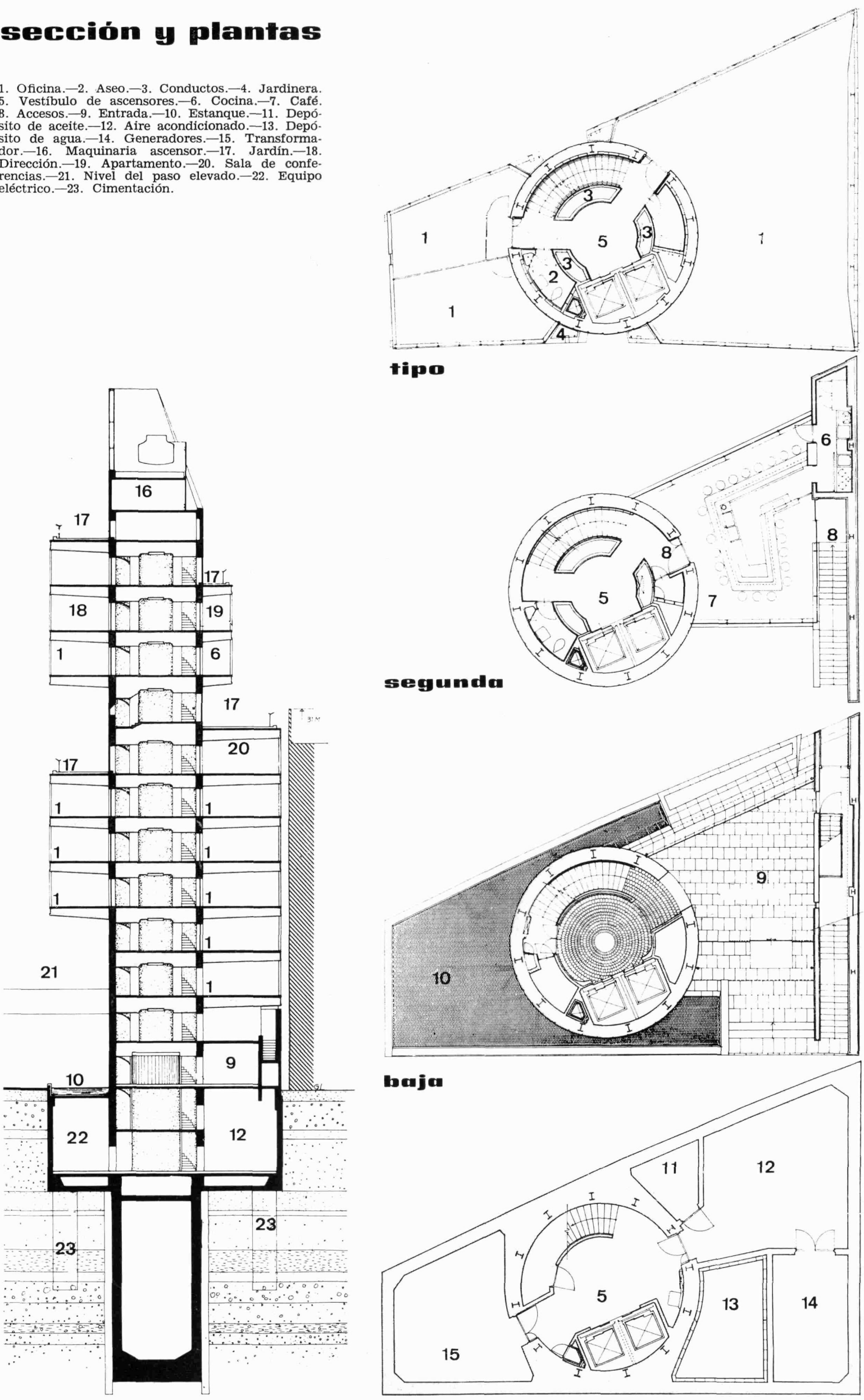

tripo

Segumale
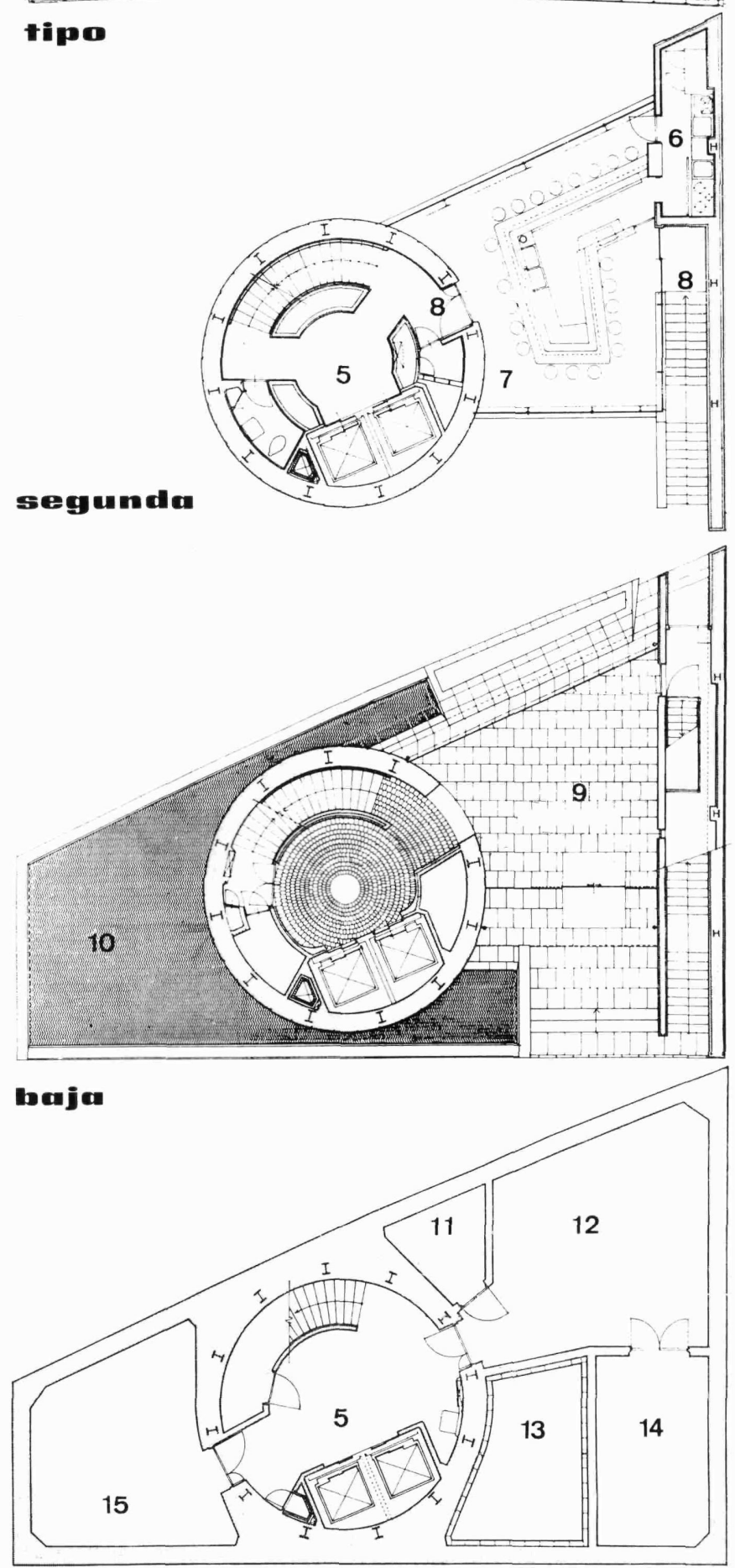


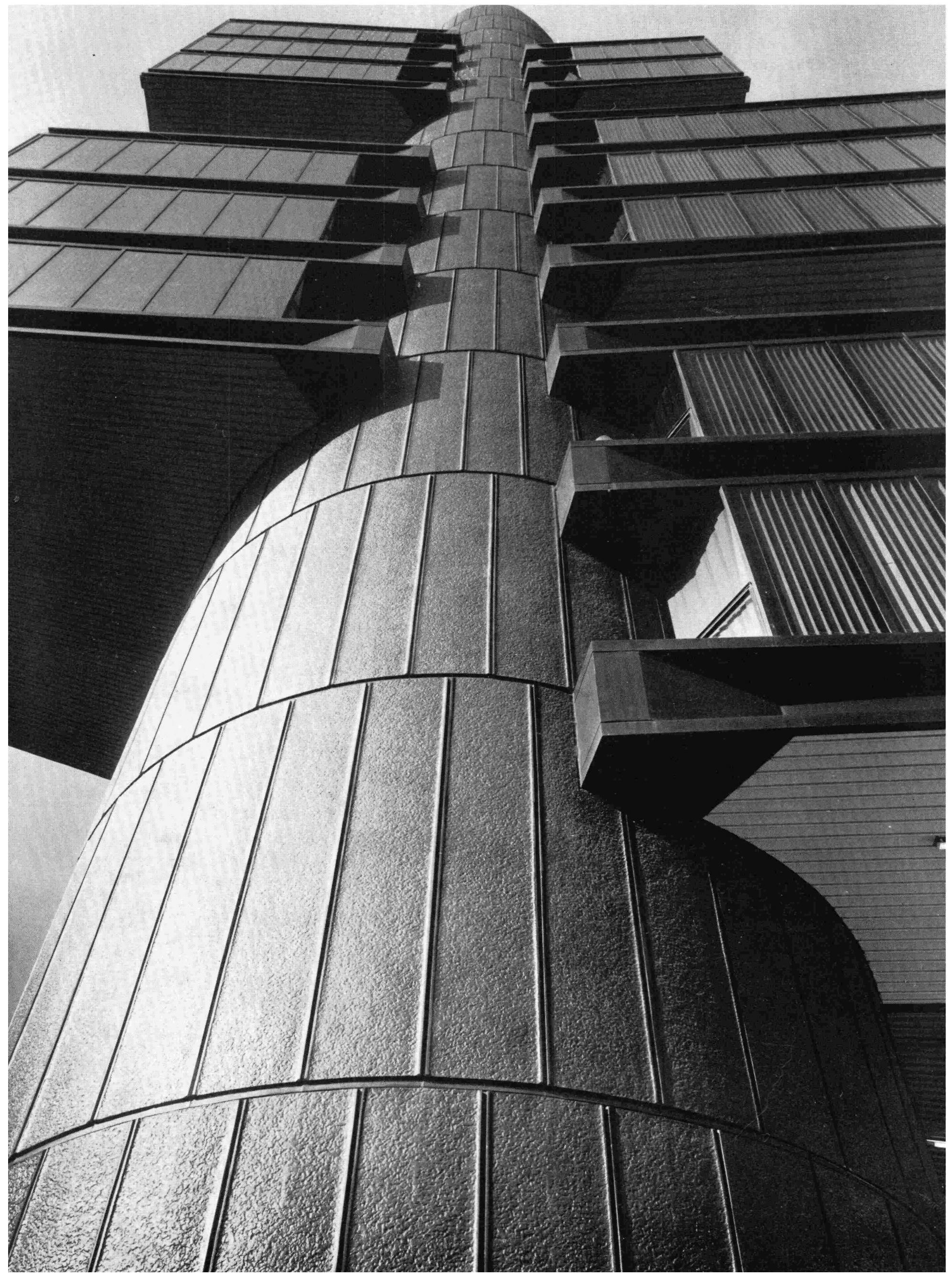

(C) Consejo Superior de Investigaciones Científicas Licencia Creative Commons 3.0 España (by-nc) 
Al concebirlo, Kenzo Tange trató de expresar no solamente las funciones arquitectónicas de la edificación, sino también los elementos urbanísticos que la integran, en el conglomerado urbano de Ginza.

Entre estos elementos figura: el acentuar y ordenar la velocidad que se desarrolla a su alrededor; el simbolizar y señalizar con una expresión característica la entrada al distrito de Ginza, y el mantener una cierta unidad con el resto de las edificaciones vecinas.

Frente a este edificio pasa el Shinkauzen, el tren más veloz del mundo; y en la autopista, los automóviles que se separan del tráfico pesado de Tokyo para acelerar su carrera hacia el aeropuerto internacional; alzándose el edificio como un pivote capaz de soportar esta imagen de velocidad.

La razón de haber sido considerado como elemento señalizador de la entrada a Ginza, está fundada en que la columna definidora de espacio es tradicional en la arquitectura japonesa desde tiempos prehistóricos; así lo podemos observar en las casas labriegas (minka), en las casas en donde se celebra la ceremonia del té (chashitsu) y en las casas ordinarias (en la columna de Tokonoma).

Si nos fijamos en otras obras del arquitecto Tange, por ejemplo en el proyecto de Skopje o en el centro de comunicaciones de Yamanashi, comprobaremos el sentido urbanístico de estas columnas; su pensamiento de estructurar las ciudades por medio de poderosas columnas circulares, que contengan los distintos servicios organizados a base de una serie de plantas horizontales, creadoras de grandes espacios libres que permitan una flexibilidad en su distribución.



\section{Maison de ritet de diffusion de journal de Shizuokra-Tokgo}

Kenzo Tange, architecte, et collaborateurs

Structure: Laboratoire Shigeru Aoki

Cet édifice se compose d'un sous-sol et de douze étages organisés autour d'un axe vertical, constitué par une colonne centrale - de $7,70 \mathrm{~m}$ de diamètre et $57 \mathrm{~m}$ de hauteur-, d’une ossature mixte: métallique et en béton armé.

Malgré les dimensions extrêmement réduites du terrain, l'édifice se détache sur l'ensemble urbain et sert de point de repère aux voyageurs qui arrivent au centre de Tokyo par les autoroutes ou par le superexpress Shinkauzen (le plus rapide du monde).

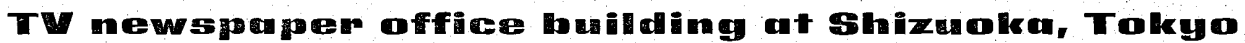

Kenzo Tange, architect, and collaborators

Structure: Shigeru Aoki Laboratory

This building has a central shaft of $7.70 \mathrm{~m}$ diameter and $5 \mathrm{~m}$ height, made of steel and reinforced concrete, and it involves a basement and 12 floors.

In spite of the small area of the site this building has become an outstanding feature of the urban landscape, and serves as a reference point to visitors to Tokyo who arrive along the motor roads or in the high speed Shinkauzen train, reputedly the fastest in the world.

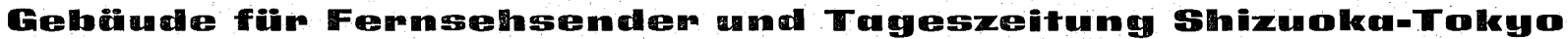

Kenzo Tanger, Architekt, und Mitarbeiter

Struktur: Laboratorium Shigeru Aoki

Das Gebäude ist um eine vertikale Achse herum angelegt, welche aus einer Zentralsäule -7,70 im Durchmesser und $57 \mathrm{~m}$ hoch- besteht auf der Grundlage einer gemischten Struktur - aus Metall und Stahlbeton-. Das Gebäude umfasst: Kellergeschoss und zwölf Obergeschosse.

Trotz der äusserst beschränkten Dimensionen des Grundstücks hebt sich das Gebäude vom übrigen Stadtbild ab und dient dem Reisenden, der über eine der Autobahnen oder mit dem Superexpress Shinkauzen (der schnellste Zug der Welt) in das Zentrum Tokios gelangt, als Richtpunkt. 